\title{
A mathematical framework for analyzing wild tomato root architecture
}

\author{
Arjun Chandrasekhar*1 and Magdalena M Julkowska ${ }^{2,3}$ \\ ${ }^{1}$ Department of Computer Science, University of Pittsburgh \\ ${ }^{2}$ Boyce-Thompson Institute \\ ${ }^{3}$ Center for Desert Agriculture, King Abdullah University for Science and Technology, \\ Jeddah, Saudi Arabia
}

\begin{abstract}
The root architecture of wild tomato, Solanum pimpinellifolium, can be viewed as a network connecting the main root to various lateral roots. Several constraints have been proposed on the structure of such biological networks, including minimizing the total amount of wire necessary for constructing the root architecture (wiring cost), and minimizing the distances (and by extension, resource transport time) between the base of the main root and the lateral roots (conduction delay). For a given set of lateral root tip locations, these two objectives compete with each other - optimizing one results in poorer performance on the other - raising the question how well S. pimpinellifolium root architectures balance this network design trade-off in a distributed manner. Here, we describe how well S. pimpinellifolium roots resolve this trade-off using the theory of Pareto optimality. We describe a mathematical model for characterizing the network structure and design trade-offs governing the structure of S. pimpinellifolium root architecture. We demonstrate that $S$. pimpinellifolium arbors construct architectures that are more optimal than would be expected by chance. Finally, we use this framework to quantify structural differences between arbors grown in the presence of salt stress, classify arbors into four distinct architectural ideotypes, and test for heritability of variation in root architecture structure.
\end{abstract}

\section{Introduction}

Root architecture in plants is driven by the development of lateral roots that branch off of the main root [1]. Our goal is to derive a model that explains the principles governing branching patterns in $S$. pimpinellifolium root architecture. Root architecture design that affords efficient resource transport is crucial to the overall productivity of the plant [2]; however, it can be advantageous to minimize the amount of material required to construct arbors due to environmental constraints such as resource limitations [3], soil compaction [4], growth environment orientation [5], and salt stress [6]. Prior work models plant arbors as a weighted graph, and uses the theory of Pareto optimality to resolve the trade-off between minimizing resource transport delay and minimizing material cost $[7,8,9]$. We extend prior work by applying a model developed for Arabidopsis to quantify design trade-offs in S. pimpinellifolium.

Root architecture is highly plastic, showing adaptability to the environments and stress conditions such as salt stress [10]. These salt-induced changes in root architecture were previously used to identify genetic components contributing to overall stress-resilience [6]. Through screening S. pimpinellifolium, which is stress-resilient relative to cultivated tomato, we aim to identify novel strategies that contribute to $S$. pimpinellifolium resilience. Through modeling and analyzing network topology, we attempt to gain further insight into network efficiency and adaptability to stress conditions.

\footnotetext{
*to whom correspondence should be addressed: arjunc@pitt.edu
} 
In addition to observing high stress-resilience, we find that S. pimpinellifolium arbors visually classified into four distinct architectural ideotypes: "Christmas Tree", "Telephone Pole", "Droopy Telephone Pole", and "Broomstick" (Fig 1A). However, a preliminary analysis revealed that this classification scheme is not associated with root structure variation according to traditional physical features (Fig 1B-D). Our goal was to study alternate methods for quantifying network topology, to test whether our qualitative classification scheme captures meaningful variation in root architecture.

Our manuscript is organized as follows. First, we define a graph-theoretic model S. pimpinellifolium arbors that formalizes the objectives of wiring cost and conduction delay. We demonstrate how to resolve the trade-off between these two objectives using the theory of Pareto optimality. We use this framework to investigate how well S. pimpinellifolium arbors optimize wiring cost and conduction delay, and infer whether these objectives are significant drivers of root architecture topology. We then investigate variation in how arbors prioritize objectives. We test whether arbors prioritize objectives in the presence of a salt stressor; we propose a scheme for classifying arbors into four architectural ideotypes, and use network design trade-offs to validate this classification scheme; finally, we explore whether genetic variation is associated with arbors prioritizing objectives differently.

\section{Related work}

In modeling and analyzing the network topology of S. pimpinellifolium, we relate our work to the Euclidean Steiner tree optimization problem [11, 12, 13]. We study the Steiner tree problem with certain constraints on the solution space; additionally our Steiner trees must balance two cost functions instead of one.

Our work is also closely related to the problem of finding a Light Approximate Shortest-Path (LAST) Tree $[14,15]$. Given a rooted graph, the goal is to find a spanning tree whose total weight is not too high while maintaining short paths from the root to each vertex. Our work attempts to solve a similar problem but allows for branch points that are not part of the original network.

\section{Materials and Methods}

Below we describe how we use graph theory, as well as the principle of Pareto optimality, to model $S$. pimpinellifolium root architecture and quantify design trade-offs.

\section{A graph-theoretic framework for studying $S$. pimpinellifolium architecture}

We model the S. pimpinellifolium root architecture as a weighted, connected, acyclic graph $A=(V, E)$. Each of the vertices $v \in V$ represents the 3-dimensional coordinate of one of the points on the root. We partition the vertices into two disjoint sets: The main root vertices $R \subset V$, and the lateral root vertices $L=V \backslash R$.

The main root vertices from a path graph - that is, they can be ordered $R=\left\{r_{1}, r_{2}, \ldots, r_{n}\right\}$ such that $\left(r_{i}, r_{i+1}\right) \in E$ for all $1 \leq i<n$. We designate $r_{1}$ as the main root base.

Each lateral root vertex has degree 1, and is directly connected to one of the main root vertices. Lateral root vertices do not connect to another lateral root segment.

For each edge $(u, v) \in E$, let the weight $w(u, v)$ be the Euclidean distance between $u$ and $v$. For each pair of points $u, v \in V$, let the distance $d(u, v)$ be the length of the (unique) path from $u$ to $v$.

Let the wiring cost be the sum of the edge lengths of all edges in the graph:

$$
W(A)=\sum_{(u, v) \in E(A)} w(u, v)
$$

Let the conduction delay be the sum of graph distances from the main root base to each lateral root: 


$$
D(A)=\sum_{l_{i} \in L} d\left(r, l_{i}\right)
$$

\section{Pareto-optimal root architecture problem}

In general, it is impossible to design a root architecture that simultaneously minimizes wiring cost and conduction delay. To resolve this trade-off, we turn to the principle of Pareto optimality [16] to define the problem of constructing an optimal arbor.

As input, we are given the following (Figure 2A):

- A set of main root points $\left\{r_{1}, r_{2}, \ldots, r_{n}\right\}$ that are all connected to each other; in other words, our network starts with the edges $\left\{\left(r_{1}, r_{2}\right),\left(r_{2}, r_{3}\right), \ldots,\left(r_{n-1}, r_{n}\right)\right\}$

- A set of lateral root tips $\left\{l_{1}, l_{2}, \ldots, l_{m}\right\}$. Initially these points are not connected to the main root.

- A value $\alpha \in[0,1]$

We then construct an architecture graph $A$ by connecting each lateral root to the main root (Figure 2A). Each lateral root may connect to one of the initial main root points, or it may connect to a midpoint on one of the edges $\left(r_{i}, r_{i+1}\right)$ (thus splitting that edge into two smaller edges). The architecture graph $A$ should minimize:

$$
\alpha \cdot D(A)+(1-\alpha) \cdot W(A)
$$

The parameter $\alpha$ determines how much the solution should prioritize one objective over the other. Different choices of $\alpha$ will yield different optimal solutions $A_{\alpha}$ that the arbor could have constructed (Figure 2A). We call these optimal architectures the Pareto front of optimal solutions.

\section{An algorithm for generating optimal root architectures}

To generate optimal root architectures, we first note lateral roots must connect directly to the main root (and not to each other); thus we can optimize the full architecture by optimizing each lateral root's connection point independently. To do so, we discretize the main root by dividing each line segment into 100 smaller line segments, thus creating several new potential connection points. For each lateral root, we consider which of connection point on the main root is optimal for that lateral root according to equation (3).

Note that this algorithm is an approximation, and can be made exact using calculus; such improvements are the subject of ongoing future work.

\section{Quantifying network design trade-offs in observed architectures}

Given an observed architecture $A$, we may use the above algorithm to compute the set of optimal root architectures: $\left\{A_{\alpha} \mid \alpha \in[0,1]\right\}$ (Figure 2A-B). Using this, we can calculate two metrics for quantifying network design trade-offs in the original observed arbor (Figure $2 \mathrm{~B}$ ):

- Pareto front distance: The L-2 distance between costs of arbor $A$ and the costs of the closest optimal tree:

$$
\min _{\alpha}\left\|(D(A), W(A))-\left(D\left(A_{\alpha}\right), W\left(A_{\alpha}\right)\right)\right\|
$$

This measures how close the original arbor came to managing an optimal trade-off between wiring cost and conduction delay.

- Pareto front location: The value of $\alpha \in[0,1]$ associated with the optimal arbor closest in cost to the observed arbor.

$$
\underset{\alpha}{\arg \min }\left\|(D(A), W(A))-\left(D\left(A_{\alpha}\right), W\left(A_{\alpha}\right)\right)\right\|
$$


The closer this value to 1 , the more strongly the arbor prioritized minimizing wiring cost at the expense of conduction delay.

Under this framework: we tested four hypotheses: 1) S. pimpinellifolium roots are closer to being Pareto optimal than would be expected by chance; 2) S. pimpinellifolium roots respond to salt stress by modifying network design trade-offs; 3) variation in Ideotype classification is associated with variation in Pareto front location; 4) Pareto front location is a heritable trait that may be driven (in part) by genetic factors.

\section{Comparing observed arbors against a null model}

To evaluate how well $S$. pimpinellifolium would be expected to optimize wiring cost and conduction delay by chance, we compared observed arbors with arbors that were randomly generated on the same set of points. To generate a random arbor, we start with the same main root segments, and same lateral root tip locations, as the original observed arbor. Each lateral root tip is then connected to a point chosen at uniform random from the set of line segments comprising the main root.

\section{Empirical Data}

The natural diversity panel 260 accessions of S. pimpinellifolium was screened for salt-stress induced changes in root system architecture. The seeds were surface sterilized using $50 \%$ bleach for 10 minutes and washed 4-6 times using sterile MiliQ water. The seeds were stratified at $4^{\circ} \mathrm{C}$ for 24 hours to improve germination. The seeds germinated on square petridishes $(12 \times 12 \mathrm{~cm})$ on $1 / 4$ Murashi-Skoog media (1.1 g MS, $5 \mathrm{~g}$ sucrose, $1 \mathrm{~g}$ M.E.S. buffer, pH $5.8 \mathrm{KOH}, 10 \mathrm{~g}$ Dashin agar). Four days after germination, the seedlings were transferred to square petridishes containing 1/4 Murashi-Skoog media for Control treatment or 1/4 MS media supplemented with $100 \mathrm{mM} \mathrm{NaCl}$ for Salt treatment. The plates were scanned every day for 7 consecutive days.

The root system architecture was quantified from the images recorded $0,1,2,3$ and 4 days after transfer to Control and Salt plates using SmartRoot [17]. The global root data was compiled and analyzed in R for lengths of main and lateral roots as well as growth rates of main root, and lateral roots. The root node data was used for calculating the Pareto optimality.

Plants were manually and qualitatively classified into one of four representative ideotype accessions (Figure 1A). The root systems where lateral roots were decreasing in length throughout the main root axis were classified as "Christmas Tree". The root systems where the lateral root was constant throughout the main root axis and where lateral roots were growing at approximately $90^{\circ}$ angle from the main root axis were classified as "Telephone Pole". The root systems similar to "Telephone Pole", but with lateral roots growing under approximately $45^{\circ}$ angle from the main root axis were classified as "Droopy Telephone Pole". The root systems where majority of the lateral roots originated at the root base were classified into "Broomstick" ideotype.

\section{Results}

\section{S. pimpinellifolium roots are Pareto optimal}

For each arbor, we computed the Pareto front of optimal architectures that the arbor could have constructed, and measured each arbor's distance to its respective Pareto front. To test whether arbors are closer to being Pareto optimal than would be expected by chance, we compared each arbor against 20 randomly generated arbors (Figure 3A). We find that S. pimpinellifolium arbors are more optimal than random trees $98.61 \%$ of the time, which is significantly higher than $50 \%$ (binomial test, $p<10^{-322}$ ). On average, the $S$. pimpinellifolium arbor is $3.15 \pm 2.60$ times closer to the Pareto front than the random arbor; this ratio is significantly higher than 1 (Welch's T-test; $p<10^{-322}$ ). We conclude that $S$. pimpinellifolium architecture optimizes wiring cost and conduction delay significantly more than would be expected by chance. This 
suggests that further study of $S$. pimpinellifolium may yield new insights into design of distributed network optimization algorithms.

\section{Salt stressor affects network design trade-offs}

Arbors grown in control conditions tended to prioritize optimizing wiring cost more than arbors grown in conditions with a salt stressor (Figure 3B). The average value of $\alpha$ for arbors grown in control conditions was was $0.55 \pm 0.26$, as compared to $0.50 \pm 0.28$; the difference between the two groups was statistically significant (Mann-Whitney test; $p<0.001$ ). This suggests that salt stressors may lead arbors to place extra emphasis on fast resource transport.

\section{Classification of ideotypes is associated with variation in network design trade- offs}

Finally, we find a strong association between an arbor's architectural ideotype and the degree to which an arbor prioritizes trade-offs between wiring cost and conduction delay (Figure 3C). There are significant differences in Pareto front location between different ideotype groups (Kruskal-Wallis one way ANOVA; $\left.p<10^{-28}\right)$. The Pareto front location was significantly different between all pairs of ideotype groups (Mann-Whitney test with a Bonferroni correction, $p<0.01$ in all cases). Thus, Pareto front location represents a quantitative measure that is associated with the architectural diversity that we observe upon visual inspection of $S$. pimpinellifolium arbors.

\section{Network design trade-offs are heritable}

The variation for Pareto front location was lower within a single genotype than the variation observed across individual genotypes. We calculated broad-sense heritability $H^{2}=\frac{V_{G}}{V_{P}}$, defined as proportion of variation due to genotype specific effects $V_{G}$ to the observed phenotypic variation across the genotypes $V_{P}$; values close to 1 indicate high heritability, and values close to 0 indicate low heritability [18]. Broad-sense heritability for Pareto front location was 0.6345 for root systems measured 4 days after transfer, indicating the high heritability of this trait and its potential use in forward genetic studies.

\section{Discussion}

Like many natural and engineered systems, S. pimpinellifolium root architecture must balance trade-offs between multiple competing objectives. Generally there is not a single architecture that is optimal for all possible objectives. Instead, the best the arbor can do is converge to an architecture that is Pareto optimal - meaning it is impossible to improve one objective without sacrificing performance in the other objectives. Our work proposes two objectives - minimizing wiring cost, and minimizing conduction delay - that $S$. pimpinellifolium roots seek to optimize in a Pareto optimal manner. Using graph theory and the principle of Pareto optimality, we define a framework for quantifying the optimality of a given S. pimpinellifolium root architecture. We apply this model to $S$. pimpinellifolium imaging data and find that $S$. pimpinellifolium arbors are significantly closer to being Pareto optimal for these objectives than would be expected by chance. We use Pareto front location to show that S. pimpinellifolium arbors prioritize objectives differently in the presence of a salt stressor; we find that unlike traditional network design metrics, Pareto front location quantifies S. pimpinellifolium architecture in a way that is strongly associated with the architectural diversity we observe qualitatively; and we find that Pareto front location shows a strong association with genetic variation. Overall, our work provides a framework for quantifying S. pimpinellifolium based on how and how well they manage network design trade-offs. More broadly, our work provides a blueprint for quantifying how natural and engineered systems manage trade-offs between several competing objectives. 


\section{Future directions}

Pareto front location is associated with our classification of S. pimpinellifolium arbors into architectural ideotypes. Future work involves using clustering analysis of Pareto front location to conclusively validate our chosen classification scheme (or identify the correct number of clusters). If we find evidence for the existence of our four identified ideotypes, future work will seek to find the functional basis behind each of these four structures.

In addition to formulating an exact algorithm for generating Pareto-optimal arbors, we seek to study the development process of $S$. pimpinellifolium. We will take advantage of data tracking the growth of individual arbors over time to measure how arbors prioritization of objectives changes over time, and eventually define a model for the distributed growth process used to generate Pareto optimal architectures.

Future work includes Genome Wide Association Study (GWAS), to identify underlying genetic components of Pareto front location and distance in S. pimpinellifolium roots. Through reverse genetic approaches we aim to further study genetic components of Pareto front location, their role in root architecture development and further developing better understanding on how S. pimpinellifolium plants prioritize objectives during non-stress and stress conditions.

Finally, we will expand our model to study the trade-off between not only wiring and transport efficiency, but also gravitropism and growth media exploration. Certain arbors may have a higher tendency to grow towards or away from gravitational forces in the growth medium. Furthermore, certain arbors may exhibit higher nutrient uptake at the main root, thus leading lateral roots to explore farther horizontally in order to avoid competing with the main root. We will expand our mathematical model to include these factors as extra constraints on how lateral roots may connect to the main root (while still optimizing wiring cost and conduction delay.

\section{Acknowledgements}

We thank the organizing committee for the 2021 Biological Distributed Algorithms (BDA) conference, as well as the reviewers for or BDA submission. We thank Guillame Bauchet for help with understanding the output from the plant imaging software. We thank Graham Zug, who is currently working with Dr. Chandrasekhar on mathematical optimization algorithms for constructing Pareto optimal plant arbors.

\section{Author Confirmation Statement}

AC: data curation, formal analysis, methodology, software, validation, visualization, writing (original draft preparation). MJ: conceptualization, data curation, formal analysis, investigation, methodology, visualization, writing (original draft preparation).

\section{Author Disclosure Statement}

We declare no competing interests.

\section{Funding Statement}

Dr. Julkowska's work was financed by the King Abdullah University for Science and Technology and the Boyce Thompson Institute. 
bioRxiv preprint doi: https://doi.org/10.1101/2021.08.12.456185; this version posted August 13, 2021. The copyright holder for this preprint

(which was not certified by peer review) is the author/funder, who has granted bioRxiv a license to display the preprint in perpetuity. It is made available under aCC-BY-ND 4.0 International license.

\section{${ }_{222}$ Data availability}

${ }_{223}$ We will make data available upon request. Our code for analyzing arbors and performing statistical 224 analysis can be found here https://github.com/arjunc12/Plant-Architecture. 

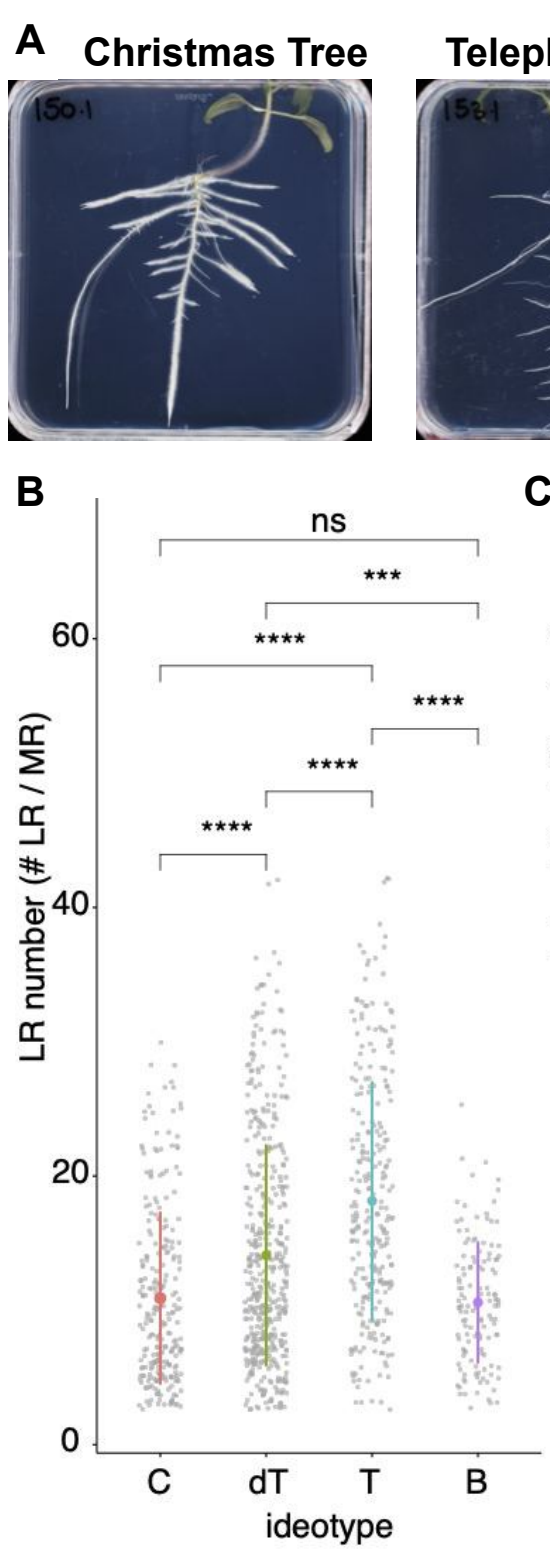

C
Telephone Pole Droopy Telephone Pole
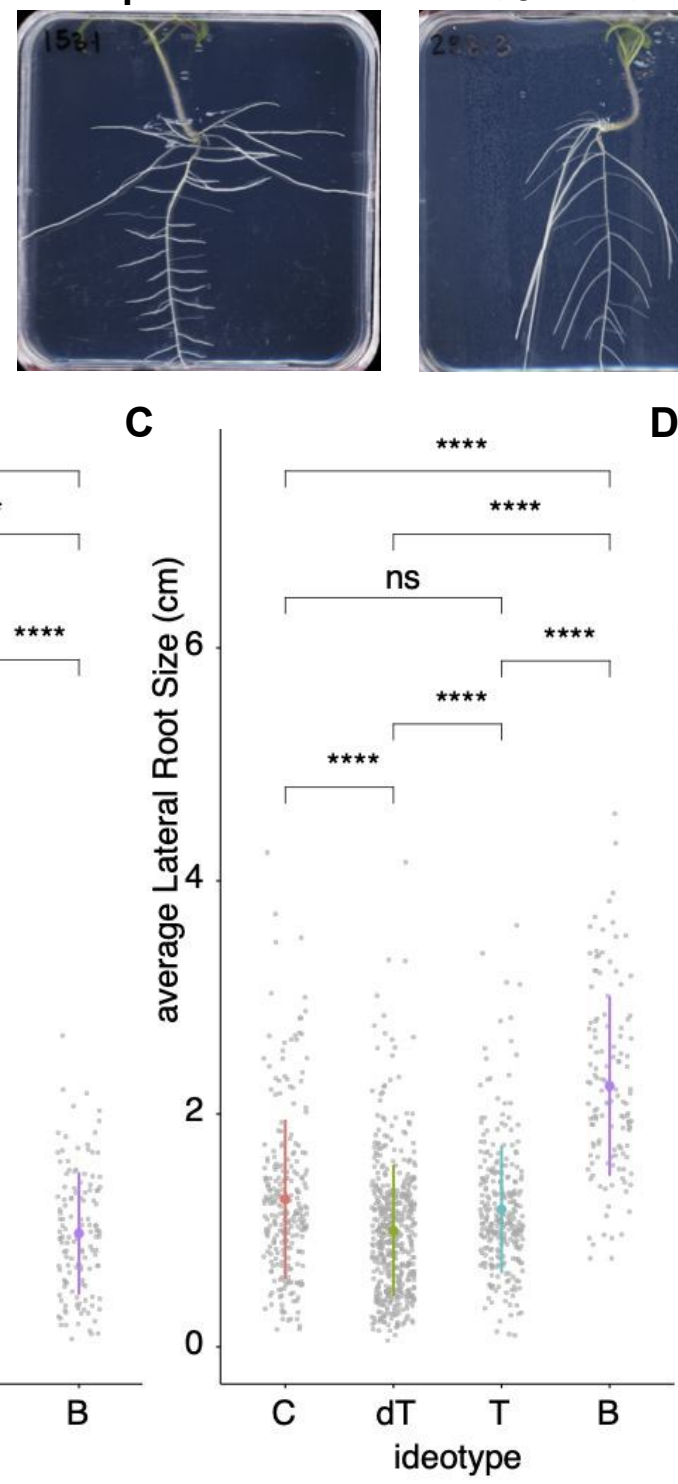
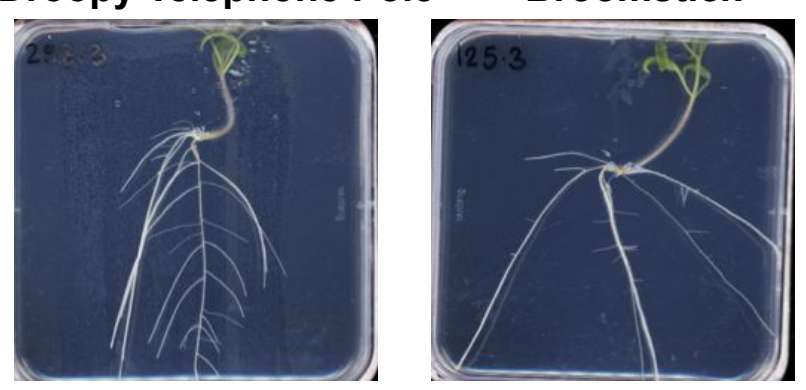

D

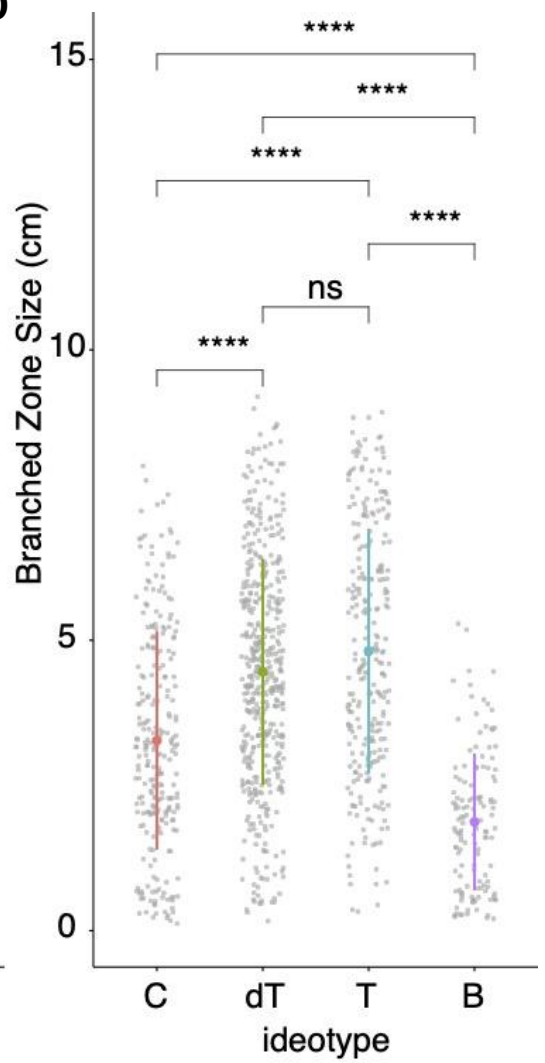

Figure 1: S. pimpinellifolium root ideotypes across the diversity panel. A) The four ideotypes of root system architecture were identified in representative accessions: M150 / LA1686 for Christmas Tree; M153 / LA1689 for telephone pole; M125 / LA1628 for broomstick; M298 / LA3160 for droopy telephone pole. The pictures represent the 12 days old seedling root system architecture grown under non-stress conditions. We compared S. pimpinellifolium ideotypes for B) number of lateral roots, C) average lateral root length and $\mathrm{D})$ length of the branched zone. The individual ideotypes are Christmas tree (C), Telephone Pole (T), Droopy Telephone Pole (dT) and Broomstick (B). The asterisks indicate significant differences between pairs of ideotypes using Tukey's honest significance test. In all cases, at least one pair of ideotypes is not detected as significantly different from each other. 

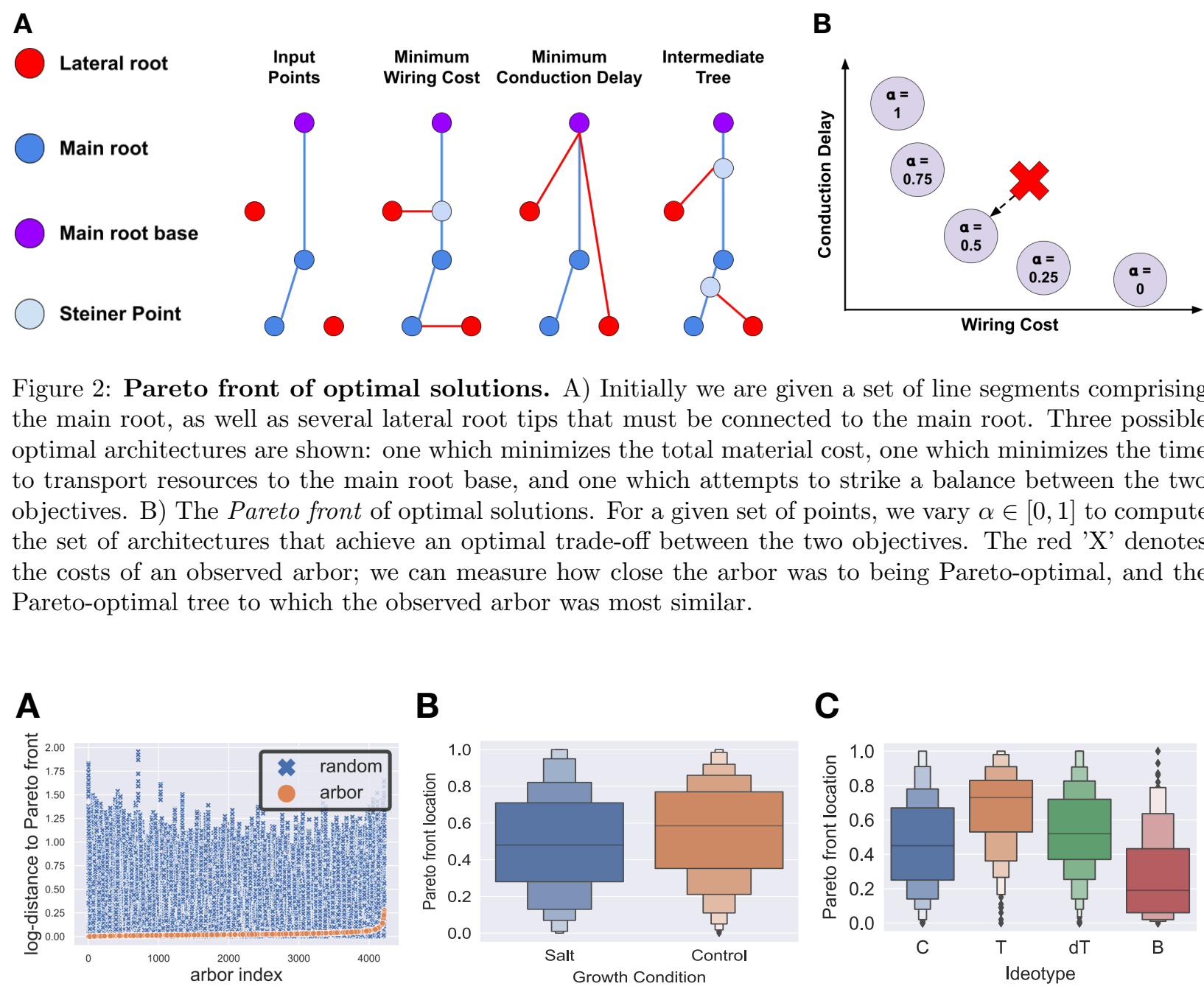

Figure 2: Pareto front of optimal solutions. A) Initially we are given a set of line segments comprising the main root, as well as several lateral root tips that must be connected to the main root. Three possible optimal architectures are shown: one which minimizes the total material cost, one which minimizes the time to transport resources to the main root base, and one which attempts to strike a balance between the two objectives. B) The Pareto front of optimal solutions. For a given set of points, we vary $\alpha \in[0,1]$ to compute the set of architectures that achieve an optimal trade-off between the two objectives. The red 'X' denotes the costs of an observed arbor; we can measure how close the arbor was to being Pareto-optimal, and the Pareto-optimal tree to which the observed arbor was most similar.

Figure 3: Analysis of Pareto front distance and location. A) For each S. pimpinellifolium arbor, we calculated the distance to the Pareto front, as well as the distance for 20 arbors that were randomly generated on the same set of main root and lateral root points. We find that S. pimpinellifolium are significantly closer to being Pareto optimal than would be expected by chance. B-C) For each arbor, we calculated its closest location on the Pareto front, a measure of how the arbor prioritized objectives. We find significant differences between how arbors grown in different conditions (B), and arbors from different ideotype classes $(\mathrm{C})$, prioritize objectives.

\section{References}

[1] C Nibau, DJ Gibbs, and JC Coates. Branching out in new directions: the control of root architecture by lateral root formation. New Phytologist, 179(3):595-614, 2008.

[2] Jonathan Lynch. Root architecture and plant productivity. Plant physiology, 109(1):7, 1995.

[3] Yao Fang Niu, Ru Shan Chai, Gu Lei Jin, Huan Wang, Cai Xian Tang, and Yong Song Zhang. Responses of root architecture development to low phosphorus availability: a review. Annals of botany, 112(2):391408, 2013. 
[4] Ying Long Chen, Jairo Palta, Jonathan Clements, Bevan Buirchell, Kadambot HM Siddique, and Zed Rengel. Root architecture alteration of narrow-leafed lupin and wheat in response to soil compaction. Field Crops Research, 165:61-70, 2014.

[5] D Chiatante, M Sarnataro, S Fusco, A Di Iorio, and GS Scippa. Modification of root morphological parameters and root architecture in seedlings of fraxinus ornus l. and spartium junceum l. growing on slopes. Plant Biosystems-An International Journal Dealing with all Aspects of Plant Biology, 137(1):47$55,2003$.

[6] Magdalena M Julkowska, Iko T Koevoets, Selena Mol, Huub Hoefsloot, Richard Feron, Mark A Tester, Joost JB Keurentjes, Arthur Korte, Michel A Haring, Gert-Jan de Boer, et al. Genetic components of root architecture remodeling in response to salt stress. The Plant Cell, 29(12):3198-3213, 2017.

[7] Adam Conn, Ullas V Pedmale, Joanne Chory, and Saket Navlakha. High-resolution laser scanning reveals plant architectures that reflect universal network design principles. Cell systems, 5(1):53-62, 2017.

[8] Adam Conn, Ullas V Pedmale, Joanne Chory, Charles F Stevens, and Saket Navlakha. A statistical description of plant shoot architecture. Current Biology, 27(14):2078-2088, 2017.

[9] Adam Conn, Arjun Chandrasekhar, Martin van Rongen, Ottoline Leyser, Joanne Chory, and Saket Navlakha. Network trade-offs and homeostasis in arabidopsis shoot architectures. PLoS computational biology, 15(9):e1007325, 2019.

[10] Magdalena M. Julkowska, Huub C.J. Hoefsloot, Selena Mol, Richard Feron, Gert-Jan de Boer, Michel A. Haring, and Christa Testerink. Capturing arabidopsis root architecture dynamics with root-fit reveals diversity in responses to salinity. Plant Physiology, 166(3):1387-1402, 2014.

[11] Sanjeev Arora. Polynomial time approximation schemes for euclidean tsp and other geometric problems. In Foundations of Computer Science, 1996. Proceedings., 37th Annual Symposium on, pages 2-11. IEEE, 1996.

[12] Marek Karpinski and Alexander Zelikovsky. New approximation algorithms for the steiner tree problems. Journal of Combinatorial Optimization, 1(1):47-65, 1997.

[13] Ajit Agrawal, Philip Klein, and R Ravi. When trees collide: An approximation algorithm for the generalized steiner problem on networks. SIAM Journal on Computing, 24(3):440-456, 1995.

[14] Samir Khuller, Balaji Raghavachari, and Neal Young. Balancing minimum spanning trees and shortestpath trees. Algorithmica, 14(4):305-321, 1995.

[15] Uyen Trang Nguyen and Jin Xu. Multicast routing in wireless mesh networks: Minimum cost trees or shortest path trees? IEEE Communications Magazine, 45(11), 2007.

[16] NO Da Cunha and E Polak. Constrained minimization under vector-valued criteria in finite dimensional spaces. Journal of Mathematical Analysis and Applications, 19(1):103-124, 1967.

[17] Guillaume Lobet, Loïc Pagès, and Xavier Draye. A Novel Image-Analysis Toolbox Enabling Quantitative Analysis of Root System Architecture. Plant Physiology, 157(1):29-39, 072011.

[18] J.F. Griffiths, D.T.S.R.C.L.S.R.W.W.M.G.A.J.F.G. Jeffrey H. Miller, A.J.F. Griffiths, U.S.R. Wessler, S.R. Wessler, R.C. Lewontin, W.M. Gelbart, D.T. Suzuki, J.H. Miller, and U.D.T. Suzuki. An Introduction to Genetic Analysis. W. H. Freeman, 2005. 